\title{
Hybrid Coherent and Frequency-Shifted-Reference Ultrawideband Radio
}

\author{
Huaping Liu*, Member, IEEE, Andreas F. Molisch ${ }^{\dagger}$, Fellow, IEEE, Shiwei Zhao*, Dennis Goeckel ${ }^{\ddagger}$, Senior Member, IEEE, \\ Philip Orlik ${ }^{\dagger}$, Member, IEEE \\ ${ }^{*}$ School of Electrical Eng. \& Computer Science; Oregon State University, Corvallis, Oregon, USA \\ $\dagger$ Mitsubishi Electric Research Lab, Cambridge, Massachusetts, USA \\ $\ddagger$ Electrical and Computer Engineering; University of Massachusetts, Amherst, Massachusetts, USA
}

\begin{abstract}
Ultrawideband communications often occur in heterogeneous networks where different receivers have different complexity and energy consumption requirements. In this case it is desirable to have a modulation scheme that works well with coherent receivers as well as simpler receivers, namely transmitted-reference (TR) receivers. In particular, we consider a TR scheme that employs slightly frequency-shifted reference (FSR) signals [15] and thus avoids one of the main drawbacks of conventional TR schemes, namely the need to implement a delay line. We propose and analyze a modulation scheme that works well with both FSR receivers (where it has the same performance as conventional TR modulation), and coherent receivers. Coherent receivers receiving conventional TR modulation suffer a $3 \mathrm{~dB}$ penalty, because they cannot make use of the energy invested into the reference pulse. Our proposed scheme avoids this drawback by including a data preprocessor that can be viewed as a nonsystematic rate- $1 / 2$ convolutional code with a high constraint length. These codes give $1.5 \mathrm{~dB}$ gain over our previously proposed constraint-length-two systematic codes at a BER of $1 \times 10^{-4}$ in 802.15.3a CM4 multipath fading channels.
\end{abstract}

Index Terms-Pulsed ultrawideband, frequency-shifted reference, coherent rake, hybrid modulation.

\section{INTRODUCTION}

One of the key challenges for pulsed ultrawideband (UWB) systems [1]-[4] is the construction of low-cost receivers that work well in multipath environments. In impulse radio, each symbol is represented by a series of pulses. In multipath environments, pulses suffer delay dispersion, and their energy has to be collected by Rake receivers or equivalent structures [5]. Coherent Rake receivers that combine all resolvable multipath components (MPCs) [6] are optimum; however, tracking, estimating, and combining a large number of MPCs (e.g., tens or even hundreds in indoor environments [7], [8]), or even a subset of all the resolvable paths [9], result in complex receivers. Consequently, simpler alternatives, in particular transmitted-reference (TR) receivers, have drawn significant attention in recent years [10]-[14].

In conventional TR systems, the symbol is represented by a series of pulse doublets, where each doublet consists of two pulses separated by a fixed delay: the first, unmodulated, pulse serves as a reference pulse; the second pulse is data modulated and is referred to as the data pulse. The received signal can

A. F. Molisch is also at Department of Electroscience, Lund University, Sweden. simply be demodulated by correlating it with a delayed version of itself, and integrating the resulting signal. The main problem for implementing TR receivers is the delay unit, which must handle wideband analog signals and must be precise, making it difficult to build in low-power integrated fashion [15], [16]. In order to avoid the delay unit, an alternative, called slightly frequency-shifted reference (FSR), was recently proposed by one of us [15]. In this scheme, the pulse doublet does not consist of pulses that are offset in the delay domain, but rather in the frequency domain. A receiver thus only has to implement a mixer, and not a delay line, and is thus much easier to build; furthermore such a receiver can actually perform slightly better than the conventional TR receiver over both additive white Gaussian noise (AWGN) and multipath fading channels [15].

Devices in a UWB network often have different cost/complexity/performance requirements. For example, some UWB devices may be battery-operated and thus require a simple, energy-saving receiver, while other devices may be connected to a power supply and thus can use a high-complexity, power hungry receiver that offers better communication range [17]. The resulting heterogeneous network requires a "universal" modulation method compatible with different types of receivers such as coherent Rake and FSR or TR receivers. Technically, it is possible to demodulate FSR signals with a coherent receiver, by simply "throwing away" the reference pulses. However, this implies a $3 \mathrm{~dB}$ signal energy penalty compared to a system that is designed to use coherent receivers only. On the other hand, signals designed for coherent receivers (i.e., those without reference pulses) obviously cannot be demodulated by a FSR receiver. In [18], we proposed a hybrid modulation scheme that enables efficient reception by both coherent and TR receivers. The key idea is to make the "reference pulse" information bearing, without modifying the phase relationship between the reference pulse and data pulse. This makes sure that the energy in the reference pulse is not "wasted" for the coherent receiver, and recovers the $3 \mathrm{~dB}$ loss by "normal" TR signaling. Furthermore, the information in the reference pulse is made dependent on the previous information symbol, which introduces memory into the modulation, and leads to further performance gain for the coherent receiver.

In the current paper, we show how our hybrid modulation 
scheme can be modified to enable coherent rake receivers and FSR receivers in the same wireless network, and present several modifications that further improve the performance. In particular, our contributions are:

- we propose a hybrid modulation scheme that works with coherent and FSR receivers.

- we propose a modified encoding structure. The data preprocessor in the basic hybrid scheme proposed in [18] can be viewed as a rate- $1 / 2$ systematic convolutional code with a constraint length two. We show in this paper that the systematic code can be extended into a nonsystematic convolutional code with longer constraint lengths that gives a higher coding gain, while the desired properties between the data pulse and reference pulse are still maintained for FSR receivers.

- we derive analytical bounds for the coding gain of the new preprocessing structure.

- we show that a frequency shift proposed in [15] can be reduced by a factor of two, improving performance, and allowing for the transmission of higher data rates.

- we analyze the resulting receiver structure and performance for the resulting UWB transmission scheme. We derive closed-form equations for the BER in multipath channels.

The remainder of the paper is organized the following way. Section II explains the improved hybrid FSR-coherent UWB scheme, and coherent and FSR receivers for the proposed hybrid modulation scheme are analyzed in Section III. Section IV presents simulation results of the proposed scheme with a coherent receiver. Comparison is made between the proposed improved hybrid FSR-coherent scheme and the basic hybrid TR-coherent scheme described in [18]. Concluding remarks are given in Section V.

\section{HYBRID FSR-COHERENT SYSTEM WITH IMPROVED DATA PREPROCESSOR}

\section{A. FSR transmitter}

We consider a time-hopping impulse radio system, where each bit interval $T_{b}$ is partitioned into $N_{f}$ frames, each having a duration $T_{f}\left(T_{b}=N_{f} T_{f}\right)$. Let us define a basic template waveform for the transmission of a bit

$$
u(t)=d_{j} \sum_{j=0}^{N_{f}-1} p\left(t-j T_{f}-c_{j} T_{c}\right)
$$

where $p(t)$ is the basic UWB pulse shape normalized so that $\int_{-\infty}^{\infty} p^{2}(t) d t=1 / N_{f}$. The elements of the pseudorandom sequence $c_{j}$, which determines the position of the pulse within each frame, are uniformly distributed integers between 0 and $T_{f} / T_{c}$, where $T_{c}$ is the chip duration. The elements of the pseudorandom sequence $d_{j}$ are taken from $\{-1,+1\}$, and effect a randomization of the polarities, which is useful for smoothing the transmit spectrum and enhancing the multiuser separation. For ease of notation, we henceforth omit the $d_{j}$, since they do not influence the operation of the TR receiver (where their impacts on reference pulse and data pulse cancel out) or the coherent receiver (where the receiver cancels its effect by multiplying the received signal with the sequence $\left.d_{j}\right)$.

We furthermore define waveforms for the reference and data signals (assuming equal energies)

$$
\begin{aligned}
& x_{r}(t)=\sum_{i=-\infty}^{\infty} \sqrt{E_{b} / 2} s_{r}[i] u\left(t-i T_{b}\right) \\
& x_{d}(t)=\sum_{i=-\infty}^{\infty} \sqrt{E_{b} / 2} s_{d}[i] u\left(t-i T_{b}\right) .
\end{aligned}
$$

The data streams $s_{r}[i]$ and $s_{d}[i]$ modulating the reference and data signals will be discussed in Sec. II-D.

In an FSR scheme, the total transmitted waveform $x(t)$ is

$$
\begin{aligned}
x(t) & =x_{r}(t)+x_{d}(t) \sqrt{2} \cos \left(2 \pi f_{0} t\right) \\
& =\sum_{i=-\infty}^{\infty} \sqrt{\frac{E_{b}}{2}}\left\{s_{r}[i]+\sqrt{2} s_{d}[i] \cos \left(2 \pi f_{0} t\right)\right\} u\left(t-i T_{b}\right)
\end{aligned}
$$

where $f_{0}$ is the shift of the center frequency of the reference pulses relative to that of the data pulses.

In order to allow simple demodulation, it is essential that $f_{0}$ be chosen such that the first and second term of the r.h.s. of Eq. (3) are orthogonal. At the same time, $f_{0}$ should be chosen to be as small as possible; this ensures that the reference signal and the (frequency-shifted) data signal "see" the same propagation channel even in frequency-selective channels. In the following, we derive the minimum frequency spacing. Without loss of generality, let us focus on the first bit interval, i.e., $i=0$.

$$
\begin{aligned}
& \int_{0}^{T_{b}} x_{r}(t) x_{d}(t) \cos \left(2 \pi f_{0} t\right) d t \\
= & \frac{E_{b}}{2} s_{r}[0] s_{d}[0] \int_{0}^{T_{b}} \sum_{j=0}^{N_{f}-1} p^{2}\left(t-j T_{f}-c_{j} T_{f}\right) \cos \left(2 \pi f_{0} t\right) d t \\
\approx & \frac{E_{b}}{2} s_{r}[0] s_{d}[0] \sum_{j=0}^{N_{f}-1} \cos \left(2 \pi f_{0} j T_{f}\right) \int_{0}^{T_{b}} p^{2}\left(t-j T_{f}\right) d t \\
= & \frac{E_{b}}{2 N_{f}} s_{r}[0] s_{d}[0] \sum_{j=0}^{N_{f}-1} \cos \left(2 \pi f_{0} j T_{f}\right)=0
\end{aligned}
$$

where the approximation is obtained by considering that $p(t-$ $\left.j T_{f}\right)$ is a very narrow pulse, compared with a bit interval $T_{b}$, located at $t=j T_{f}$. When $N_{f}$ is large and the $N_{f}$ pulses are equally spaced over $T_{b}$, choosing $f_{0}=\frac{1}{2 T_{b}}$ will imply that the summands $\cos \left(2 \pi f_{0} j T_{f}\right)$ in the last line are the uniform samples of the sinusoidal signal $\cos \left(2 \pi f_{0} t\right)$ over the first half of its fundamental period from 0 to $T_{b}$, which is approximately equal to zero.

This choice of the frequency-shift reduces the difference in the center frequencies of the data pulse and reference pulse by a half compared with the suggested value of $f_{0}=\frac{1}{T_{b}}$ in [15]. 


\section{B. Channel}

The standardized 802.15.3a channel model [7] describes the channel impulse response as

$$
h(t)=\sum_{l=0}^{L-1} h_{l} \delta\left(t-\tau_{l}\right)
$$

where $L$ is the total number of multipath components, $h_{l}$ is the channel fading coefficient for the $l$-th path, $\tau_{l}$ is the arrival time of the $l$-th path relative to the first path ( $\tau_{0}=0$ assumed), and $\delta(t)$ is the Dirac delta function. The channel gain $h_{l}$ is modeled as $h_{l}=\lambda_{l} \beta_{l}$, where $\lambda_{l}$ takes on the values of -1 or 1 with equal probability and $\beta_{l}$ has a lognormal distribution [7]. Since multipath components tend to arrive in clusters [7], $\tau_{l}$ in (5) is expressed as $\tau_{l}=\mu_{c}+\nu_{m, c}$, where $\mu_{c}$ is the delay of the $c$-th cluster that the $l$-th path falls in, $\nu_{m, c}$ is the delay (relative to $\mu_{c}$ ) of the $m$-th multipath component in the $c$-th cluster. The relative power of the $l$-th path to the first path can be expressed as $E\left\{\left|h_{l}\right|^{2}\right\}=E\left\{\left|h_{0}\right|^{2}\right\} e^{-\mu_{c} / \Gamma} e^{-\nu_{m, c} / \gamma}$, where $E\{\cdot\}$ denotes expectation, $\Gamma$ is the cluster decay factor, and $\gamma$ is the ray decay factor. Note that, different from common baseband models of narrow-band systems, $h_{l}$ is real-valued.

\section{FSR receiver}

The FSR receiver is similar to the one described in [15] and is shown in Fig. 1 for convenience. The received signal is bandpass-filtered ${ }^{1}$, multiplied with a frequency-shifted version of itself, and integrated.

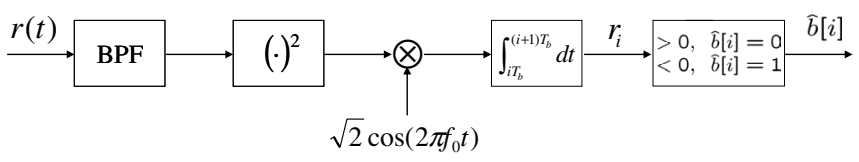

Fig. 1. FSR receiver for the proposed hybrid FSR-coherent scheme.

Using an analysis similar to [15], we can show that for the $i$-th bit, the integrator output $r_{i}$ in the absence of noise can be expressed as

$$
\begin{aligned}
r_{i}= & \int_{i T_{b}}^{(i+1) T_{b}}\left(\sqrt{\frac{E_{b}}{2}}\left\{s_{r}[i]+\sqrt{2} s_{d}[i] \cos \left(2 \pi f_{0} t\right)\right\}\right. \\
& \left.u\left(t-i T_{b}\right)\right)^{2} \sqrt{2} \cos \left(2 \pi f_{0} t\right) d t \\
= & E_{b} s_{r}[i] s_{d}[i]
\end{aligned}
$$

where the second equation can be derived using the relationship given in (4).

\footnotetext{
${ }^{1}$ The bandpass filter (BPF) has bandwidth $B$, which is chosen to be larger than the signal's $10 \mathrm{~dB}$ bandwidth, to avoid severe, filter-induced distortion to the signal. For simplicity of analysis, it is also assumed that the BPF is ideal and its bandwidth $B$ is an integer multiple of $1 /\left(2 T_{b}\right)$. The filtered noise is no longer white, but its autocorrelation can be very narrow in time due to the ultra-wide filter bandwidth $B$.
}

\section{Improved data processor}

In the conventional FSR scheme, the reference bits are fixed, i.e., $s_{r}[i]=1$. However, coherent receivers can achieve better performance if the reference signal also carries information; of course, it is essential to retain a signaling structure that allows demodulation by an FSR receiver.

The proposed hybrid scheme is shown in Fig. 2, where the 'reference' bit $b_{r}[i]$ and 'data' bit $b_{d}[i]$ can be considered as the outputs of a rate- $1 / 2$ convolutional encoder. In the related scheme of [18], we proposed a convolutional encoder with constraint length 2 , resulting in a minimum free distance of $\sqrt{6}$. We now investigate a new encoding scheme with a constraint length three and code generator polynomial $\left[1 \oplus D \oplus D^{2}, 1 \oplus D^{2}\right]$, where $\oplus$ denotes modulotwo addition. Note that the 'reference' bit is not really a reference bit as in a conventional TR system; rather, it is one of the two output bits of the data preprocessor, i.e., the rate$1 / 2$ convolutional encoder. Obviously, a coherent receiver can detect the transmitted bits by demodulating both the reference bit and the data bit, and then applying a Viterbi decoder to recover the original information bits.

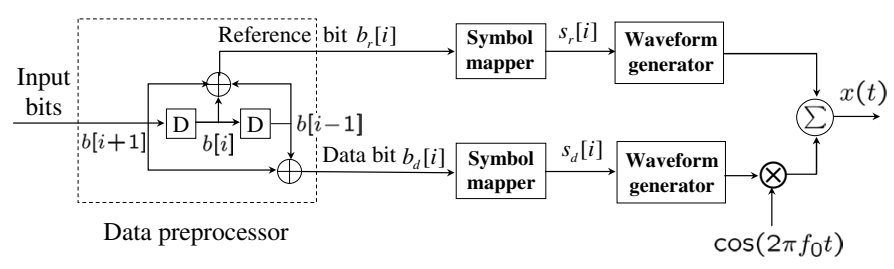

Fig. 2. Block diagram of the slightly frequency-shifted-reference hybrid system transmitter.

This encoder structure must satisfy certain conditions so that FSR receivers can detect the transmitted signal as well. Table I shows the polarities of the modulated reference and data waveforms, and their phase/polarity difference for all eight combinations of three consecutive input bits. As seen from the table, the phase difference of the pair of pulses - the reference and data pulses - solely depends on input information bit $b[i]$; therefore, an FSR receiver can also demodulate the received signals.

TABLE I

INPUT-OUTPUT COMBINATIONS OF DATA PREPROCESSOR DESIGNED AS A RATE- $1 / 2$ CONVOLUTIONAL CODE WITH CONSTRAINT LENGTH THREE

\begin{tabular}{||c|c|c||c|c||c|}
\hline$b[i+1]$ & $b[i]$ & $b[i-1]$ & $s_{r}[i]$ & $s_{d}[i]$ & $\begin{array}{c}\text { Phase difference } \\
\text { between } \\
s_{r}[i] \text { and } s_{d}[i]\end{array}$ \\
\hline \hline 0 & 0 & 0 & -1 & -1 & $0^{o}$ \\
\hline 0 & 0 & 1 & +1 & +1 & $0^{o}$ \\
\hline 0 & 1 & 0 & +1 & -1 & $180^{\circ}$ \\
\hline 0 & 1 & 1 & -1 & +1 & $180^{o}$ \\
\hline 1 & 0 & 0 & +1 & +1 & $0^{\circ}$ \\
\hline 1 & 0 & 1 & -1 & -1 & $0^{o}$ \\
\hline 1 & 1 & 0 & -1 & +1 & $180^{\circ}$ \\
\hline 1 & 1 & 1 & +1 & -1 & $180^{o}$ \\
\hline
\end{tabular}


The $i$-th data bit and the $i$-th reference bit are expressed as

$$
\begin{aligned}
& b_{d}[i]=b[i+1] \oplus b[i-1] \\
& b_{r}[i]=b[i+1] \oplus b[i] \oplus b[i-1] .
\end{aligned}
$$

For bipolar signaling, the symbol mapper simply performs the following mapping: $b_{r}[i]=0 / 1 \rightarrow s_{r}[i]=-1 / 1 ; b_{d}[i]=$ $0 / 1 \rightarrow s_{d}[i]=-1 / 1$.

For coherent rake receivers, the data preprocessor is effectively a rate- $1 / 2$ convolutional encoder with a constraint length three. The minimum Euclidean distance is $\sqrt{10 \varepsilon}$, resulting in a $4 \mathrm{~dB}$ coding gain, which is $2.22 \mathrm{~dB}$ higher than the basic hybrid TR-coherent scheme in [18].

The proposed data preprocessor can be further extended to a rate- $1 / 2$ convolutional encoder with a longer constraint length. For example, the convolutional code with generator polynomial $\left[1 \oplus D \oplus D^{2} \oplus D^{3}, 1 \oplus D^{2} \oplus D^{3}\right]$ is a good choice with a constraint length four. The relationship among the input bits, the reference bit, and the data bit for this preprocessor is shown in Table II. The minimum Euclidean distance with this preprocessor becomes $\sqrt{12 \varepsilon}$, which results in an additional $0.8 \mathrm{~dB}$ coding gain over the preprocessor shown in Fig. 2, or $3 \mathrm{~dB}$ coding gain over the scheme in [18].

TABLE II

INPUT-OUTPUT COMBINATIONS OF DATA PREPROCESSOR DESIGNED AS A RATE- $1 / 2$ CONVOLUTIONAL CODE WITH CONSTRAINT LENGTH FOUR

\begin{tabular}{||c|c|c|c||c|c||c|}
\hline$b[i+1]$ & $b[i]$ & $b[i-1]$ & $b[i-2]$ & $s_{r}[i]$ & $s_{d}[i]$ & $\begin{array}{c}\text { Phase difference } \\
\text { between } \\
s_{r}[i] \text { and } s_{d}[i]\end{array}$ \\
\hline \hline 0 & 0 & 0 & 0 & -1 & -1 & $0^{\circ}$ \\
\hline 0 & 0 & 0 & 1 & +1 & +1 & $0^{o}$ \\
\hline 0 & 0 & 1 & 0 & +1 & +1 & $0^{\circ}$ \\
\hline 0 & 0 & 1 & 1 & -1 & -1 & $0^{\circ}$ \\
\hline 0 & 1 & 0 & 0 & +1 & -1 & $180^{\circ}$ \\
\hline 0 & 1 & 0 & 1 & -1 & +1 & $180^{\circ}$ \\
\hline 0 & 1 & 1 & 0 & -1 & +1 & $180^{\circ}$ \\
\hline 0 & 1 & 1 & 1 & +1 & -1 & $180^{\circ}$ \\
\hline 1 & 0 & 0 & 0 & +1 & +1 & $0^{\circ}$ \\
\hline 1 & 0 & 0 & 1 & -1 & -1 & $0^{\circ}$ \\
\hline 1 & 0 & 1 & 0 & -1 & -1 & $0^{\circ}$ \\
\hline 1 & 0 & 1 & 1 & +1 & +1 & $0^{\circ}$ \\
\hline 1 & 1 & 0 & 0 & -1 & +1 & $180^{\circ}$ \\
\hline 1 & 1 & 0 & 1 & +1 & -1 & $180^{\circ}$ \\
\hline 1 & 1 & 1 & 0 & +1 & -1 & $180^{\circ}$ \\
\hline 1 & 1 & 1 & 1 & -1 & +1 & $180^{\circ}$ \\
\hline
\end{tabular}

For even higher constraint lengths, it becomes difficult to find good codes with the maximum, or near maximum, free distance while the phase relationship between the reference pulse and data pulse required by FSR receivers is still maintained. A longer constraint length would generally result in a higher coding gain at the expense of a higher decoding complexity. Thus, system complexity-performance tradeoff can be made flexible by choosing an appropriate preprocessor structure.

\section{PERFORMANCE ANALYSIS}

\section{A. FSR receiver in multipath}

Let the received signal at the BPF output be expressed as $r(t)=x(t) * h(t)+n(t)$, where $h(t)$ is given in (5), $x(t)$ is given in (3), * denotes convolution, and $n(t)$ is the zero-mean Gaussian noise with a one-sided spectral density $N_{0}$. Again using an analysis similar to [15], the BER over multipath channels can be written as

$$
P_{\mathrm{FSR}}=E_{h}\left[Q\left(\frac{E_{b} \sum_{l=0}^{L-1} h_{l}^{2} \cos \left(2 \pi f_{0} \tau_{l}\right)}{\sqrt{\frac{5}{2} E_{b} N_{0} \sum_{l=0}^{L-1} h_{l}^{2}+T_{b} N_{0}^{2} B}}\right)\right]
$$

where $E_{h}[\cdot]$ denotes the expectation over the set of channel coefficients $\left\{h_{l}\right\}$. While a numerical evaluation of the expectation operator has been used in the past, we derive in the following a closed-form expression.

In the following, we assume that $f_{0} \tau_{l} \ll 1$, which is fulfilled for signaling at low to medium data rates. Let $\gamma=$ $\sum_{l=0}^{L-1} h_{l}^{2}=\sum_{l=0}^{L-1} \beta_{l}^{2}$. Note that $\left\{\beta_{l}\right\}$ are independent of one another. Since $\beta_{l}$ is a lognormal random variable $(\mathrm{RV}), \beta_{l}^{2}$ is also a lognormal RV. Thus, $\gamma$ is a sum of independent lognormal RVs. Let $\beta_{l}=e^{v_{l}}$, where $v_{l}$ is a normal RV, i.e., $v_{l} \sim N\left(\mu_{v_{l}}, \sigma_{v_{l}}^{2}\right)$. The $k$-th moment of $\beta_{l}$ is given as

$$
E\left\{\beta_{l}^{k}\right\}=e^{k \mu_{v_{l}}+k^{2} \sigma_{v_{l}}^{2} / 2} .
$$

Although an exact closed-form expression of the pdf of a sum of independent lognormal RVs does not exist, such a sum can be approximated by another lognormal RV [20]. The parameters $\mu$ and $\sigma$ of this random variable can be obtained from the following set of nonlinear equations [19]:

$$
\begin{aligned}
& \sum_{i=1}^{N_{G}} \frac{w_{i}}{\sqrt{\pi}} \exp \left[-s_{m} \exp \left(\frac{\sqrt{2} \sigma a_{i}+\mu}{\xi}\right)\right] \\
= & \prod_{i=1}^{K} \widehat{\Psi}_{X}\left(s_{m} ; \mu_{i}, \sigma_{i}\right),
\end{aligned}
$$

where $m=1,2$ and

$$
\widehat{\Psi}_{X}(s ; \mu, \sigma)=\sum_{i=1}^{K} \frac{w_{i}}{\sqrt{\pi}} \exp \left[-s \exp \left(\frac{\sqrt{2} \sigma a_{i}+\mu}{\xi}\right)\right]
$$

and the weights, $w_{i}$, and abscissas, $a_{i}$, of the Gaussian quadrature for different orders, $N_{G}$, are tabulated in standard mathematical references. The parameters $s_{1}$ and $s_{2}$ are the arguments of the moment-generating function at which the exact distribution of the sum of lognormal variables should match the distribution of the equivalent lognormal variable. As we are mainly interested in high SNRs, small values of $s_{1}$ and $s_{2}$ should be used, e.g., $s_{1}=1$ and $s_{2}=0.2$ [19].

The approximated pdf of $\gamma$ is given as

$$
f(\gamma)=\frac{1}{\gamma \sqrt{2 \pi \sigma_{z}^{2}}} \exp \left[-\frac{\left(\ln (\gamma)-\mu_{v}\right)^{2}}{2 \sigma_{v}^{2}}\right] .
$$

The average BER can be calculated by averaging the conditional BER $P_{\mathrm{FSR}}(\gamma)=Q\left(\frac{E_{b} \gamma}{\sqrt{5 E_{b} N_{0} \gamma / 2+T_{b} N_{0}^{2} B}}\right)$ over $f(\gamma)$ as

$$
P_{\mathrm{FSR}}=\int_{0}^{\infty} P_{\mathrm{FSR}}(\gamma) f(\gamma) d \gamma
$$




\section{B. Coherent receiver}

The coherent receiver for the hybrid FSR scheme is shown in Fig. 3. The received signal is first passed through a BPF (see Section II-C) and then correlated with $\sum_{l=0}^{L-1} h_{l} u\left(t-i T_{b}-\tau_{l}\right)$. This correlator requires the knowledge of $\left\{h_{l}\right\}$ and $\left\{\tau_{l}\right\}$; thus, it is equivalently a coherent rake receiver. The correlator output is processed further to generate the estimate of both the reference bit and the data bit. For the data bit, the correlator output is integrated over each bit interval; for the reference bit, the the correlator output is first multiplied by $\sqrt{2} \cos \left(2 \pi f_{0} t\right)$ and then integrated over each bit interval. Finally, both estimates for the data bits $\left\{b_{d}[i]\right\}$ and reference bits $\left\{b_{r}[i]\right\}$ are applied for Viterbi decoding, yielding the estimated input bits $\{\hat{b}[i]\}$.

Because of the convolutional decoding involved in the coherent receiver for the hybrid scheme, the exact BER expression is difficult to obtain. However, a union bound can be derived in the following manner. First, let $T(D, I)=$ $\sum_{i, j} n(i, j) I^{i} D^{j}$ be the convolutional code transfer function, where $n(i, j)$ is the number of paths with $i$ input 1 's and $j$ output 1's that diverge from the all zero's path and remerge with it later. Assume that $h(t)$ does not vary appreciable between such a divergence and re-merge for the shorter paths that dominate the error performance (i.e., no temporal diversity is achieved). Then, note that: (1) the proposed codes are linear, and (2) the noise in one branch of the receiver is independent of the noise in the other branch of the receiver (since they are uncorrelated and jointly Gaussian). Then, applying a union bound:

$$
P_{b} \leq \sum_{i, j} i n(i, j) P\left(\underline{0}_{j} \rightarrow \underline{1}_{j}\right)
$$

where $\underline{0}_{j}$ and $\underline{1}_{j}$ are vectors of $j$ 's and $j$ 1's respectively, and $P\left(\underline{0}_{j} \rightarrow \underline{1}_{j}\right)$ is the probability of deciding the latter when the former was sent. Continuing, $P_{b}$ is upper bounded as:

$$
\begin{aligned}
& \leq \sum_{i, j} i n(i, j) E_{h}\left[P\left(\underline{0}_{j} \rightarrow \underline{1}_{j} \mid h\right)\right] \\
& =\sum_{i, j} i n(i, j) E_{h}\left[Q\left(\sqrt{\frac{E_{b} j}{N_{0}} \sum_{l-0}^{L-1} h_{l}^{2}}\right)\right] \\
& =\sum_{i, j} i n(i, j) \int_{0}^{\infty} Q\left(\sqrt{\frac{E_{b} j}{N_{0}} \gamma}\right) f(\gamma) d \gamma \\
& =\int_{0}^{\infty}\left[\sum_{i, j} i n(i, j) \frac{1}{\pi} \int_{0}^{\frac{\pi}{2}} e^{-\frac{E_{b}}{2 N_{0}} \frac{j \gamma}{\sin ^{2} \theta}} d \theta\right] f(\gamma) d \gamma \\
& =\frac{1}{\pi} \int_{0}^{\infty} \int_{0}^{\frac{\pi}{2}} \sum_{i, j} i n(i, j)\left[e^{-\frac{E_{b}}{2 N_{0}} \frac{\gamma}{\sin ^{2} \theta}}\right]^{j} f(\gamma) d \theta d \gamma \\
& =\left.\frac{1}{\pi} \int_{0}^{\infty} \int_{0}^{\frac{\pi}{2}} \frac{\partial}{\partial I} T(D, I)\right|_{I=1, D=\exp \left(-\frac{E_{b}}{2 N_{0}} \frac{\gamma}{\sin ^{2} \theta}\right)} f(\gamma) d \theta d \gamma .
\end{aligned}
$$

For small numbers of states, $T(D, I)$ can be found by hand, but this becomes unwieldy for larger numbers of states. In such cases, Appendix 6A1 of [22] gives an efficient method that can be extended to the case here.

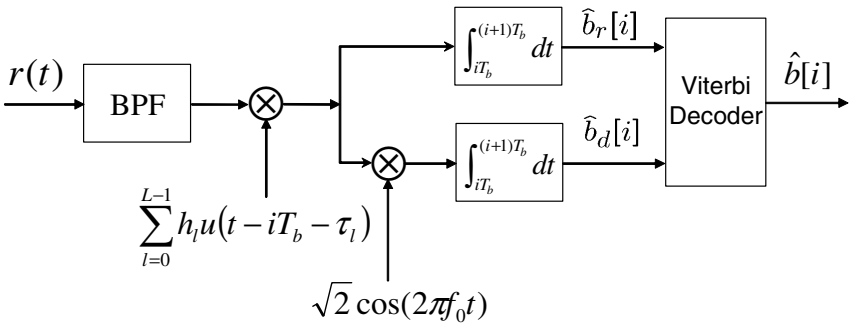

Fig. 3. Coherent receiver for the proposed hybrid FSR-coherent scheme.

\section{NUMERICAL RESULTS}

Since the performance of the FSR receiver for the proposed hybrid scheme is the same as the FSR scheme in [15], we only focus on simulating the performance with the coherent rake receiver. In all simulations, a carrier-modulated, truncated root-raised-cosine pulse with a roll-off factor 0.25 is used as the UWB pulse shape $p(t)$. The $10-\mathrm{dB}$ signal bandwidth is $1 \mathrm{GHz}$. We adopt channel models from the IEEE 802.15.3a [23] with a large delay spread (CM4) and assume perfect knowledge of the channel fading coefficients and delays. We simulate a low-data-rate system operating at $1 \mathrm{Mbps}$ and the number of pulses to represent a bit is $N_{f}=10$. For each bit, the receiver combines ten strongest paths using maximal ratio combining (MRC). This is achieved by setting the delays $\tau_{l}$ of the correlator to those of the ten strongest paths. The integrator outputs are fed into a Viterbi decoder, which approximates the maximum likelihood sequence detector. The simulation also assumes a quasi-static fading model, i.e., the channel coefficients and delays do not change over each packet duration but change independently over different packets. The total number of packets is 200 , each with 256 bits; thus, the BER values are effectively the average over 200 realizations of the channel coefficients.

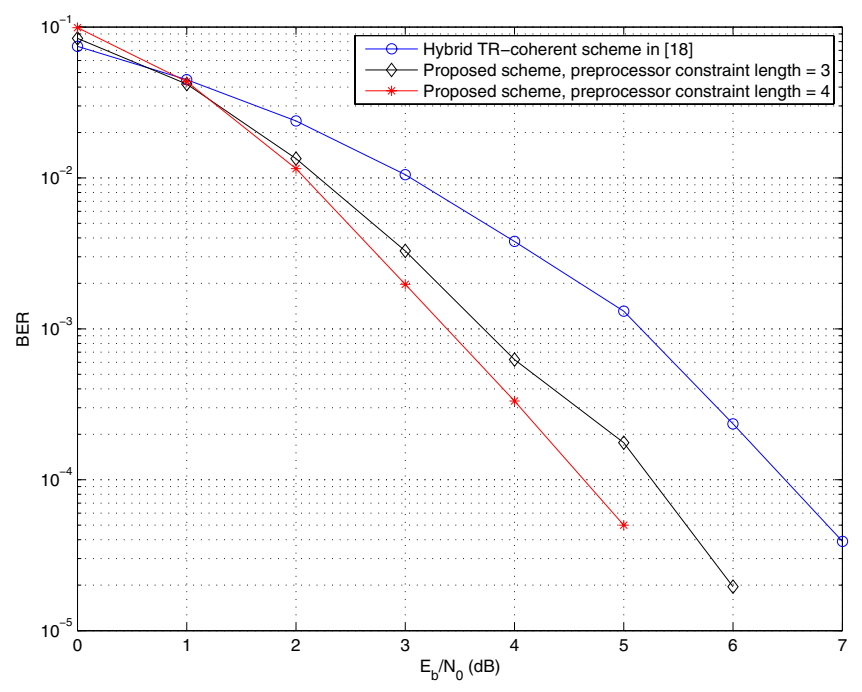

Fig. 4. BER versus $E_{b} / N_{0}$ curves of the proposed hybrid system with a coherent rake receiver operating over an AWGN channel. Performance of the hybrid TR scheme proposed in [18] is provided for comparison.

Fig. 4 shows the performance of the proposed hybrid 
FSR-coherent system with coherent detection and soft input Viterbi decoding over AWGN channels. BER performances of the proposed hybrid scheme with the data preprocessor of constraint length three and constraint length four over multipath fading channels modeled by IEEE 802.15.3a CM4 are shown in Fig. 5. For comparison, the performance of the hybrid TR-coherent system with a coherent receiver in [18] in the same environment is included in the two figures. It is observed that with the data preprocessor with a constraint length 4 , the proposed scheme achieves about a $1.5 \mathrm{~dB}$ gain at a BER of $1 \times 10^{-4}$ over the scheme in [18]; with the data preprocessor with a constraint length 3 , the proposed scheme achieves about a $1.2 \mathrm{~dB}$ gain over the scheme in [18].



Fig. 5. BER versus $E_{B} / N_{0}$ curves of the proposed hybrid system with a coherent rake receiver operating over an IEEE 802.15.3a multipath fading channel modeled by CM4. Performance of the hybrid TR scheme proposed in [18] is provided for comparison.

\section{CONCLUSION}

We have proposed a hybrid UWB modulation scheme that allows reception by both coherent and FSR receivers, and derived closed-form equations and bounds for its performance. For coherent receivers, we introduce a data preprocessing scheme where both the reference signal and data signal in the proposed hybrid FSR-coherent system are generated from the input bits by the data preprocessor. This allows the coherent receiver to exploit not only the energy invested in the reference pulses, but also the memory introduced by the preprocessor for improved performance. The proposed scheme extends the preprocessor in [18] from an effective convolutional encoder with a constraint length two to three or more, improving the performance by more than $2 \mathrm{~dB}$. Compared with coherent detection of the conventional uncoded TR scheme, the proposed scheme not only recovers the $3 \mathrm{~dB}$ loss due to the energy wasted in the reference signals, but also achieves an additional $4 \mathrm{~dB}$ coding gain. We also derived analytical bounds for the performance of the decoder in multipath environments.

\section{REFERENCES}

[1] M. G. diBenedetto, T. Kaiser, A. F. Molisch, I. Oppermann, C. Politano, and D. Porcino (eds.), UWB communications systems: a comprehensive overview, Hindawi, 2006.

[2] R. C. Qiu, H. Liu, and X. Shen, "Ultra-wideband for multiple access communications," IEEE Comm. Mag., vol. 43, pp. 80-87, Feb. 2005.

[3] L. Yang and G. B. Giannakis, "Ultra-wideband communications - an idea whose time has come," IEEE Signal Processing Magazine, vol. 21, pp. 26-54, 2004.

[4] M. Z. Win and R. A. Scholtz, "Impulse radio: how it works," IEEE Commun. Lett., vol. 2, pp. 36-38, Feb. 1998.

[5] A. F. Molisch, Wireless Communications, IEEE-Press - Wiley, 2005.

[6] M. Z. Win and R. A. Scholtz, "On the energy capture of ultrawide bandwidth signals in dense multipath environments," IEEE Commun. Lett., vol. 2, no. 9, pp. 245-247, Sep. 1998.

[7] A. F. Molisch, J. R. Foerster, and M. Pendergrass, "Channel models for ultrawideband personal area networks," IEEE Wireless Commun., vol. 10, pp. 14-21, Dec. 2003.

[8] A. F. Molisch et al. "A comprehensive model for ultrawideband propagation channels," IEEE Trans. Antennas Prop., vol. 54, pp. 3151-3166, 2006.

[9] D. Cassioli, M. Z. Win, A. F. Molisch, and F. Vatelaro, "Performance of selective Rake reception in a realistic UWB channel," in Proc. ICC 2002, Apr. 2002, pp. 763-767.

[10] R. T. Hoctor and H. W. Tomlinson, "An overview of delay-hopped, transmitted-reference RF communications," Technical Information Series, GE Research and Development Center, pp. 1-29, Jan. 2002.

[11] J. D. Choi and W. E. Stark, "Performance of ultra-wideband communications with suboptimal receiver in multipath channels," IEEE J. Select Areas Commun., vol. 20, no. 9, pp. 1754-1766, Dec. 2002.

[12] K. Witrisal, G. Leus, M. Pausini, and C. Krall, "Equivalent system model and equalization of differential impulse radio UWB systems," IEEE $J$. Selected Areas Commun., vol. 23, pp. 1851-1862, 2005.

[13] T. Q. S. Quek and M. Z. Win, "Analysis of UWB transmitted-reference communication systems in dense multipath channels," IEEE J. Selected Areas Commun., vol. 23, pp. 1863-1874, 2005.

[14] S. Zhao, H. Liu, and Z. Tian, "Decision directed autocorrelation receivers for pulsed ultra-wideband systems," IEEE Trans. Wireless Commun., vol. 5, pp. 2175-2184, Aug. 2006.

[15] D. L. Goeckel and Q. Zhang, "Slightly frequency-shifted reference ultrawideband (UWB) radio," IEEE Trans. Commun., Mar. 2007.

[16] W. Gifford and M. Z. Win, "On transmitted-reference UWB communications," in Proc. Asilomar Conf. Signals, Systems, Com., 2004.

[17] IEEE 802.15 Working Group, "Alternative PHY layer amendment for personal area networks," IEEE 802.15.4a standard, Mar. 2007.

[18] S. Zhao, P. Orlik, A. F. Molisch, H. Liu, and J. Zhang, "Hybrid Ultrawideband Modulations Compatible for Both Coherent and TransmitReference Receivers," IEEE Trans. Wireless Commun., vol. 6, no. 7, pp. 2551-2559, July 2007.

[19] N. B. Mehta, A. F. Molisch, J. Wu, and J. Zhang, "Approximating the sum of correlated lognormal or lognormal-Rice random variables," in Proc. IEEE ICC'06, Jun. 2006, pp. 1605-1610.

[20] H. Liu, "Error performance of a pulse amplitude and position modulated ultra-wideband system in lognormal fading channels," IEEE Commun. Lett., vol. 7, pp.531-533, Nov. 2003.

[21] F. Rajwani and N. C. Beaulieu, "Simplified bit error rate analysis of PAPM-UWB with MRC and EGC in lognormal fading channel," in Proc. IEEE ICC, May 2005, pp. 2886-2889.

[22] S. Wilson, Digital Modulation and Coding, Upper Saddle River: Prentice-Hall, 1996.

[23] A. F. Molisch, K. Balakrishnan, C.-C. Chong, S. Emami, A. Fort, J. Karedal, J. Kunisch, H. Schantz, U. Schuster, and K. Siwiak, "IEEE 802.15.4a channel model - final report," Document IEEE 802.15-040662-02-004a, 2005. 